\title{
Stoichiometric unfolding of bovine serum albumin by surfactant, as revealed from HPLC/SAXS with online observation of UV-Vis absorption and refractive index
}

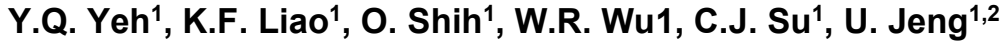 \\ ${ }^{1}$ National Synchrotron Radiation Research Center, Hsinchu 30076, Taiwan \\ ${ }^{2}$ Department of Chemical Engineering, National Tsing Hua University, Hsinchu 30013, Taiwan
}

yeh.yq@nsrrc.org.tw

Detergents are commonly used to disrupt noncovalent interactions of proteins, leading to detergent-protein complex or stabilized recombinant proteins. In past, many methods have been used to investigate conformational changes of proteins and protein-detergent complexes to understand their interactions, polarity and stability in varied detergent concentrations. The local structure of such protein/detergent complex could be resolved by spectroscopies; however, resolving the corresponding stoichiometric protein unfolding conformation requires separating the effects contributed by the coexisted protein/detergent complex and SDS micelles in the solution.

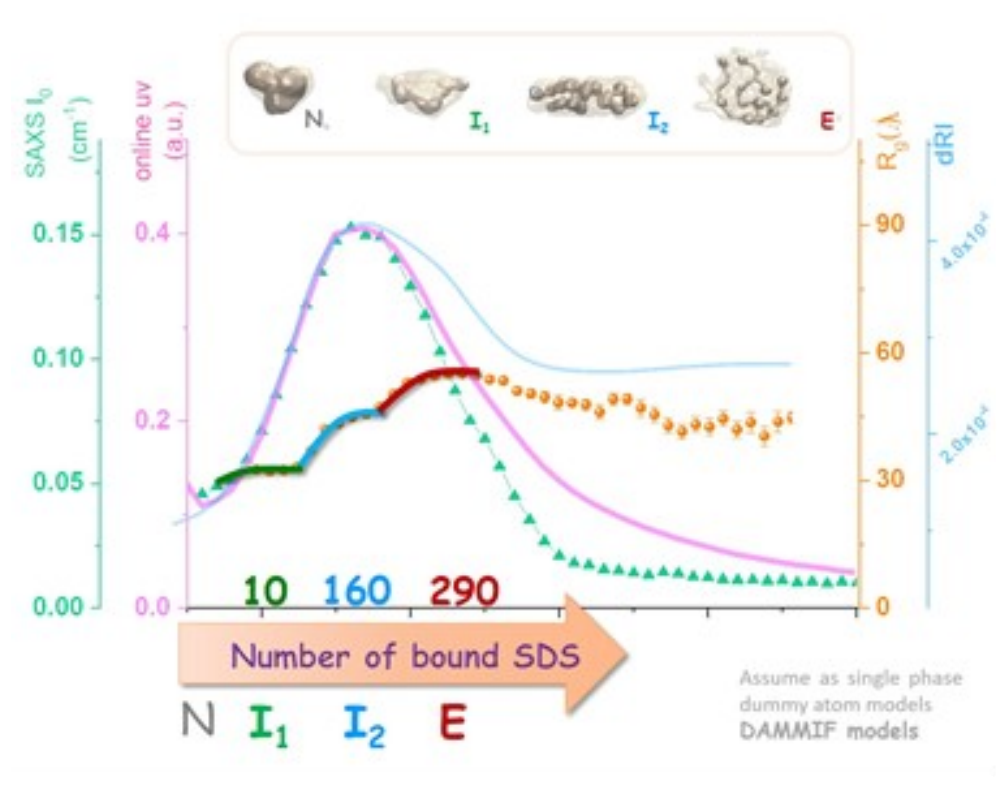

Figure 1. Rg and I0 profiles extracted from the SAXS data measured along the chromatogram IUV280 nm of the BSA/SDS solution in SDS buffer. Bottom shows the DAMMIF models of the complexes at the states indicated. (Shown models are assumed as single phase and further analysed by two-phase MD simulation.).

In this work, we show that sodium dodecyl sulfate (SDS), a frequently used surfactant in purification of membrane proteins, can bind to bovine serum albumin (BSA) for multistage unfolding. The on-line protein purification system of high performance liquid chromatography (SEC-HPLC) incorporated to the beamline 13A synchrotron BioSWAN instrument of the Taiwan Photon Source at the National Synchrotron Radiation Research Center, allows separating the scattering contributions from the BSA/SDS complexes and SDS micelles. Together with integrated observations of UV-vis absorption and refractive index (RI), we have resolved the stoichiometric unfolding conformations of BSA by SDS monomers to micelles. Offline SEC-MALS (multi angle light scattering (MALS) results are also consistent. In Figure 1, the corresponding protein-SDS association numbers along the unfolding process are determined uniquely from a combined analysis of UV-Vis absorption, refractive index, and zero-angle SAXS intensity measured in one sample elution. The q range of SAXS/WAXS profiles covers $0.007 \AA^{-1}-3 \AA^{-1}$. It was measured by Eiger 9M SAXS detector and Eiger X1M WAXS detector, moving freely inside the detector vacuum vessel. The WAXS features implied the conformational change of inter-domain motions within BSA-SDS complex.

Keywords: BSA; SDS; unfolding; BioSWAN 